\title{
Fatores psicossociais e sociodemográficos associados à depressão pós-parto: Um estudo em hospitais público e privado da cidade de São Paulo, Brasil ${ }^{1}$
}

\author{
Maria de Lima Salum e Morais \\ Secretaria de Estado de Saúde de São Paulo \\ Luiz Augusto Marcondes Fonseca \\ Vinicius Frayze David \\ Lia Matos Viegas \\ Emma Otta \\ Universidade de São Paulo
}

\begin{abstract}
Resumo
O estudo avaliou a prevalência de depressão pós-parto (DPP) e fatores associados em mulheres que deram à luz em dois hospitais da cidade de São Paulo: um público e outro privado. Foram aplicados questionários padronizados, a Escala de Depressão Pós-parto de Edimburgo (EDPE) e a Escala de Apoio Social de MOS (EAS) a 462 mulheres: 205, no hospital público e 257, no privado. Foram obtidos dados sociodemográficos, psicossociais, obstétricos e do recém-nascido (RN). Consideraram-se deprimidas mulheres com 12 ou mais pontos na EDPE, aplicada no $3^{\circ}$ ou $4^{\circ}$ mês após o parto. No hospital público, a prevalência de DPP foi de $26 \%$ e, no privado, de $9 \%$. Características dos RN foram semelhantes nas duas amostras; idade, escolaridade, número de visitas de pré-natal e de cesarianas das mães foram maiores no hospital privado. Análise de regressão envolvendo características psicossociais das participantes revelou associação positiva de DPP com depressão anterior e com frequência de conflitos com o parceiro e relação negativa com anos de escolaridade e escore de apoio social.
\end{abstract}

Palavras-chave: depressão pós-parto; prevalência; fatores de risco; hospital público; hospital privado.

\begin{abstract}
Psychosocial and sociodemographic factors associated with postpartum depression:

A study in private and public hospitals in São Paulo, Brazil. This study evaluated the prevalence of postpartum depression (PPD) and associated factors in women who gave birth at two hospitals in São Paulo City: one public and other private. It was applied standardized questionnaires, the Edinburgh Postnatal Depression Scale (EPDS), and the MOS Social Support Scale to 462 women: 205 in the public hospitals and 257, in the private one. Data collected included sociodemographic, psychosocial, obstetric and newborn (NB) characteristics. It was considered depressed those women with 12 or more points in EPDS, applied in the $3^{\text {rd }}$. or $4^{\text {th }}$. month after delivery. In the public hospital, the prevalence of PPD was $26 \%$ and in the private, $9 \%$. Characteristics of infants were similar in both samples, Mothers' age, education level, number of prenatal visits and cesarean were higher in the private hospital. Regression analysis involving psychosocial characteristics of women showed a positive association of PPD with previous occurrence of depression and frequency of conflicts with partner and negative relationship with years of schooling, and social support scores.
\end{abstract}

Keywords: postpartum depression; prevalence; risk factors; public hospital; private hospital.

\section{Resumen}

Factores psicosociales y sociodemográficos asociados con la depresión posparto: Un estudio realizado en hospital público y privado en la ciudad de São Paulo, Brasil. El estudio evaluó la prevalencia de la depresión posparto (DPP) y factores asociados en mujeres que dieron a luz en dos hospitales de la ciudad de São Paulo: un público y otro privado. Se aplicó dos instrumentos estandarizados, la escala de depresión posparto de Edimburgo (EDPE) y el Cuestionario MOS-SSS de Apoyo Social, a 462 mujeres: 205 en el hospital público y 257 en el hospital privado. Se obtuvieron datos sociodemográficos, psicosociales, obstétricos y del recién nacido (RN). Fueron consideradas deprimidas las mujeres con 12 o más puntos en la EDPE aplicada en el tercero o cuarto mes después del parto. En el hospital público, la prevalencia de DPP fue de $26 \%$ y en el privado, $9 \%$. Características de los RN fueron similares en las dos muestras; la edad, la escolaridad, el número de consultas de prenatal y de cesáreas fueron mayores en el hospital privado. El análisis de regresión implicando características psicosociales de las participantes reveló una asociación positiva de la DPP con depresión previa y con frecuencia de conflictos con el compañero y una relación negativa con los años de escolaridad y con el escore de apoyo social.

Palabras clave: depresión posparto; prevalencia; factores de riesgo; hospital público; hospital privado. 
$\mathrm{E}$ pisódios depressivos são caracterizados, de maneira geral, por rebaixamento do humor, redução de energia e da atividade, alteração na capacidade de experimentar prazer e concentração diminuída, podendo ser acompanhados por problemas de sono, diminuição da autoestima e sentimentos de culpa (Santos, Almeida, \& Souza, 2009). Segundo grande parte dos autores, a depressão pós-parto (DPP), embora possa ser desencadeada por alguns fatores etiológicos específicos do período em que ocorre (Camacho et al., 2006), apresenta as mesmas características dos demais episódios depressivos (Silva \& Botti, 2005). Seu estudo tem merecido especial atenção pelo fato de ocorrer em um período sensível e vulnerável para a mãe e seu bebê, o que a torna, também, um importante tema para a atenção em saúde. Tais características justificam o grande número de trabalhos sobre o assunto.

Não há consenso total entre os autores a respeito da duração precisa do período que se considera como depressão pós-parto: alguns afirmam que seu início ocorre logo após o nascimento do bebê [Organização Mundial da Saúde (OMS), 2007]; outros definem como DPP os episódios depressivos que iniciam a partir de duas semanas até três meses após o parto (Cantilino, Zabaldi, Sougey, \& Rennó Jr, 2010), podendo prolongar-se por semanas ou meses. A DPP diferencia-se da chamada melancolia da maternidade ou disforia puerperal, pois esse é um distúrbio de humor transitório, que costuma durar até duas semanas após o parto (Santos et al., 2009).

Diversos são os estudos nacionais e internacionais a respeito da prevalência da DPP, assim como muitos são os instrumentos utilizados para diagnosticá-la (Segre, O’Hara, Arndt, \& Stuart, 2007). O presente estudo utilizou a Escala de Depressão Pós-Parto de Edimburgo (EDPE), instrumento desenvolvido por Cox, Holden e Sagovsky (1987) para triagem de casos de DPP e validado no Brasil por Santos, Martins e Pasquali (1999) e por Santos et al. (2007). Estudos que compararam várias escalas de triagem de DPP mostram que existe uma correlação significativa entre a EDPE e a maior parte dos instrumentos destinados a avaliar sintomas de depressão (Gaynes et al.,2005; Santos et al., 2009).

Encontra-se, na literatura, uma grande variação nos índices de prevalência de DPP, em função de diversos fatores, como instrumentos de medida, condições sociais e psicológicas das díades mãe-bebê, entre outros. Halbreich e Karkun (2006), revisando 143 estudos realizados em 40 países, encontraram prevalência de DPP de zero a aproximadamente 60\%. Klainin e Arthur (2009), revisando estudos feitos em países asiáticos, relataram uma variação de 3,5\% a 63,3\%. Segre et al. (2007), estudando mulheres moradoras do estado de Iowa nos EUA, encontraram variação de 6,7 a 30,2\%. Tomemos alguns exemplos de estudos brasileiros que usaram a EDPE: Da-Silva, Moraes-Santos, Carvalho, Martins e Teixeira (1998), em estudo realizado em São Gonçalo no estado do Rio de Janeiro, constataram prevalência de $12 \%$ de mulheres com DPP; Cruz, Simões e Faisal-Cury (2005) verificaram, em mulheres atendidas em unidades do Programa da Saúde da Família na cidade de São Paulo, 37,1\% de prevalência de DPP; Rushi et al. (2007) encontraram, em Unidade Básica de Saúde situada na grande Vitória (Espírito Santo), prevalência de $39,4 \%$ de mulheres com indicativos de DPP.

Tem-se demonstrado que a grande variação de prevalência de DPP depende de uma gama de fatores de ordem sociodemográfica e psicossocial. No que se refere à influência de va- riáveis sociodemográficas sobre a prevalência de DPP, Segre et al. (2007) encontraram, como fatores preditivos, baixa renda, baixa escolaridade, baixo prestígio ocupacional, idade mais baixa, ser solteira e ter muitos filhos, sendo o nível de renda o fator mais significativo. Estudando mulheres de uma área rural da Índia, Chandran, Tharyan, Muliyil e Abraham (2002) também constataram que baixa renda é um dos importantes fatores de risco para DPP. Moraes et al. (2006), em estudo feito em Pelotas, Rio Grande do Sul, também relatam efeitos de baixa renda familiar sobre a prevalência de DPP. Segre et al. (2007) explicam o efeito de baixa renda sobre a prevalência de DPP principalmente pelo nível de estresse causado por dificuldades financeiras, das quais decorre pouco acesso a recursos de educação, saúde, alimentação, transporte e moradia.

A maior parte dos estudos realizados em diversos países confirma esses achados. Contudo, há algumas exceções que nos levam a pensar na influência conjunta de fatores culturais e socioeconômicos: Miyake, Tanaka, Sasaki e Hirota (2001), num estudo com mulheres japonesas, encontraram que emprego qualificado era fator de proteção para DPP, mas não verificaram efeito de nível de renda nem de escolaridade sobre sua incidência.

Fatores psicológicos também estão associados à ocorrência de DPP: Quelopana, Champion e Rubilar (2011), em pesquisa com uma amostra de mulheres chilenas, apontam depressão e/ou ansiedade anteriores como fatores predisponentes para o desenvolvimento de DPP. Chandran et al. (2002), estudando mulheres de uma área rural da Índia, e Topiwala, Hothi e Ebmeier (2012), referindo-se a inquérito feito no Reino Unido, encontraram que um dos principais fatores de risco pra a incidência de DPP é uma história prévia de depressão. Klainin e Arthur (2009), revisando estudos de DPP em populações asiáticas, também assinalam a influência de sintomas depressivos anteriores sobre a incidência de DPP. Aliane, Mamede e Furtado (2011), em revisão sistemática sobre fatores de risco associados à DPP, constataram, de forma consonante com os trabalhos citados, que os fatores mais frequentes foram aqueles de ordem psicológica ou psiquiátrica, socioeconômico/cultural e de suporte social.

Em contrapartida, pesquisas que procuraram relações entre dados antropométricos das crianças e depressão materna não têm sustentado a influência de fatores psicossociais e sociodemográficos. Ertel, Koenen, Rich-Edwards e Gillman (2010) não encontraram correlação entre depressão materna e altura reduzida numa amostra de díades mãe-criança nos Estados Unidos da América. Grote et al. (2010) confirmaram achados no mesmo sentido numa coorte europeia. Moraes et al. (2006), estudando díades em Pelotas, não encontraram influência da depressão materna sobre o peso do bebê ao nascer.

Por sua vez, a maioria dos trabalhos que pesquisaram a relação entre depressão materna e variáveis que dizem respeito a apoio social constataram que a falta de apoio do parceiro, de familiares e de amigos é importante fator de risco para o desenvolvimento de DPP. Chandran et al. (2002), em estudo com mulheres indianas, constataram que dificuldades de relacionamento com pais e sogras é um dos fatores de risco para DPP. Cruz et al. (2005), testando a associação de diversos fatores à DPP numa amostra de mães paulistanas, encontraram que apenas o suporte por parte do marido apresentou efeito protetor em relação à depressão da mulher. Melo Jr. et al. (2012) também encontraram em amostra brasileira relação entre risco de DPP e falta de apoio familiar . 
No que diz respeito ao relacionamento conjugal e ao apoio social, Mcvey e Tuohy (2007) desenvolveram um estudo com mulheres britânicas em que procuraram a associação desses fatores com o que chamaram de subescalas heterogêneas da Escala de Depressão Pós-Parto de Edimburgo. São elas: sintomas inespecíficos de depressão, ansiedade e anedonia. Os autores administraram a EDPE a mulheres britânicas e analisaram a relação das três subescalas da EDPE com a qualidade do relacionamento conjugal e com escalas de avaliação de apoio social, constatando diferentes relações entre essas variáveis a as subescalas. Os autores alertam para a possibilidade de não se considerar a EDPE como instrumento unidimensional.

Por essa razão, neste estudo - que objetiva avaliar o efeito de diversos fatores sociodemográficos (nível socioeconômico - representado pelo hospital do parto -, escolaridade, número de filhos e engajamento em trabalho) e psicossociais (viver com o companheiro, qualidade de relacionamento com ele, apoio social e relato de depressão anterior) sobre a ocorrência de DPP em mulheres cujos partos ocorreram em dois hospitais da cidade de São Paulo: um público e outro privado - procedeuse, também, à análise fatorial exploratória da EDPE e analisouse em que medida os fatores sociodemográficos e psicossociais estavam associados às subescalas do instrumento.

\section{Método}

\section{Participantes}

Participaram da pesquisa 462 mulheres moradoras da cidade de São Paulo, sendo 205 usuárias do sistema público de saúde, recrutadas em Unidades Básicas de Saúde da zona oeste da cidade, com parto realizado em hospital público e 257, cujo parto ocorreu em hospital privado da zona sudoeste da cidade. A maior parte das gestantes usuárias do serviço público era das classes baixa e média baixa, enquanto as mulheres que deram à luz no hospital privado pertenciam às classes média alta e alta. Portanto, neste estudo, o hospital foi uma variável proxy de nível socioeconômico. Foram excluídas da amostra mães de gêmeos, de bebês de muito baixo peso ou com suspeita de problemas graves de saúde ao nascer. Todas as mães incluídas nos estudos tiveram, portanto, bebês considerados saudáveis.

\section{Instrumentos}

Foram aplicadas duas escalas: a Escala de Apoio Social de MOS (EAS), que avaliou o apoio social percebido pelas participantes, e a Escala de Depressão Pós-Parto de Edimburgo (EDPE), que avaliou sintomas de depressão nas mães entrevistadas. Na seção de procedimento, detalha-se o momento e modo de aplicação dos dois instrumentos.

Escala de Depressão Pós-Parto de Edimburgo (EDPE). O instrumento, já referido na introdução, é composto de 10 questões que avaliam como a mulher se sentiu na última semana. Para cada pergunta há quatro opções de resposta, sendo que a cada resposta é associada uma pontuação que varia de zero a três. A soma dos pontos das repostas resulta no escore da escala, que varia de zero a 30 pontos. Para a população brasileira, pontuações iguais ou superiores a 12 pontos são indicativas de depressão pós-parto (Santos et al., 1999). As questões da EDPE se referem a como a pessoa tem se sentido nos últimos sete dias e estão reproduzidas na Tabela 3 da seção de resultados.

Escala de Apoio Social (EAS). A escala, validada no Brasil por Griep, Chor, Faerstein, Werneck e Lopes (2005), é composta por 19 questões que avaliam o apoio social percebido pelas participantes. As questões referem-se à frequência com que a pessoa conta com alguém para lhe dar suporte. Para cada pergunta há cinco possibilidades de resposta, que variam de nunca (um ponto) até sempre (cinco pontos). A pontuação dada a cada pergunta é somada e quanto maior o escore final, maior o suporte social percebido pela informante.

Questões analisadas das entrevistas com as mães. Além das escalas padronizadas, as mães responderam a diversas questões, dentre as quais foram analisadas neste trabalho: anos de escolaridade; número de filhos; ter trabalho; presença de episódio anterior de depressão ou procura prévia de psicólogo ou psiquiatra por motivo de ansiedade ou tristeza; viver (ou não) com o companheiro; e avaliação do relacionamento conjugal como muito conflituoso, um pouco conflituoso ou nada conflituoso.

\section{Procedimento}

As participantes foram recrutadas de forma diferente, dependendo da amostra à qual pertenciam. As usuárias do hospital público foram recrutadas em três Unidades Básicas de Saúde (UBS) da zona Oeste de São Paulo. O recrutamento ocorreu durante o último trimestre de gestação, tanto diretamente na UBS, antes ou depois de uma consulta de pré-natal, quanto por meio de contato telefônico a partir do cadastro de gestantes fornecido pelas unidades de saúde. Essas mulheres responderam, no total, a três entrevistas, tendo a primeira acontecido na UBS durante o último trimestre de gestação. Nessa entrevista, coletaram-se dados gerais sobre a mãe (escolaridade, número de filhos, relação com o parceiro, episódios anteriores de depressão) e foi aplicada a Escala de Apoio Social (EAS). A segunda entrevista foi realizada até dois dias após o parto e teve como objetivo principal verificar o número de consultas de pré-natal, tipo de parto e dados a respeito do bebê recémnascido (peso, altura, APGAR e estado de saúde). A terceira entrevista ocorreu entre nove e 12 semanas após o parto, na UBS ou no laboratório do Instituto de Psicologia da Universidade em que o estudo foi realizado. Nessa ocasião, foi aplicada a Escala de Depressão Pós-Parto de Edimburgo (EDPE).

As usuárias do hospital privado foram recrutadas no hospital em que deram à luz e responderam a duas entrevistas. A primeira entrevista ocorreu até dois dias após o parto e antes da alta. O roteiro foi uma combinação da primeira e da segunda entrevistas realizadas com mulheres do sistema público de saúde, inclusive com a aplicação EAS. A segunda entrevista, feita por telefone, ocorreu entre nove e 12 semanas após o parto e teve como objetivos principais levantar alguns dados sobre o bebê (aleitamento, sono, problemas de saúde, entre outros), sua inserção na família, relacionamento do casal após o parto e avaliar se a mãe tinha sintomas de depressão pós-parto, utilizando-se a EDPE.

\section{Análise de dados}

As amostras dos hospitais público e privado foram comparadas em relação às variáveis categóricas DPP (sim/não), sexo do bebê (masculino ou feminino), presença de depressão ante- 
rior ( $\operatorname{sim} /$ não), se trabalha ( $\operatorname{sim} /$ não) e se vive com companheiro ( $\operatorname{sim} /$ não) utilizando-se o teste exato de Fischer. O teste quiquadrado foi utilizado para o tipo de parto, variável categórica com três níveis (normal, cesariana, fórceps). As variáveis peso e altura do bebê e escolaridade das mães mostraram distribuição normal e foram comparadas através do teste $t$ de Student. O escore obtido na Escala de Apoio Social mostrou distribuição não normal e utilizou-se o teste de Mann-Whitney para comparação entre os escores das duas amostras. Esse mesmo teste foi usado para as variáveis escalares APGAR do quinto minuto, número prévio de filhos e qualidade de vida conjugal.

Para investigar a influência das variáveis coletadas sobre a presença ou não de DPP, aplicou-se um modelo de regressão logística com função de ligação logito. Para a seleção das variáveis, foram utilizados os métodos de seleção Stepwise, Backward e Forward, com nível de significância de 0,15 para selecionar a variável e de 0,02 para removê-la. Também foi testada a adequabilidade do modelo através do teste de Hosmer e Lemeshow (2000). Foram selecionadas para essa análise as variáveis hospital, número prévio de filhos, escolaridade, escore na EAS, qualidade da relação com o parceiro, se vive com o companheiro, se trabalha e depressão anterior, por serem consideradas as mais relevantes em outros estudos (Aliane et al., 2011; Chandran et al., 2012; Moraes et al., 2006; Rushi et al., 2007).

Para verificar como as questões da EDPE se agrupavam em fatores, foi realizada uma análise fatorial exploratória, por meio do método de rotação VARIMAX e com o critério de Kaiser. Para realizar as análises acima mencionadas foi usado o software IBM SPSS Statisitics 19 (Norusis, 2011).

Finalmente, para testar como as variáveis hospital, número prévio de filhos, escolaridade, apoio social, qualidade da relação com o parceiro, se vive com o companheiro, se trabalha e depressão anterior influenciam nos fatores formados pelas questões da EDPE, foi utilizado um modelo de equações estruturais. Para isso, empregou-se o software de análise estatística IBM-SPSS-AMOS na versão 19 (Arbuckle, 1995) e foram consideradas as métricas de avaliação do ajuste, raiz do erro quadrático médio de aproximação (RMSEA) e valores de NFI, IFI, RFI, TLI, GFI e AGFI.

\section{Aspectos éticos}

A pesquisa seguiu as normas e princípios estabelecidos na resolução CNS 196/96, tendo seu projeto sido aprovado pelo Comitê de Ética do Hospital Universitário da USP, registrado sob $n^{\circ} 673 / 06$. Todas as participantes assinaram Termo de Consentimento Livre e Esclarecido, no qual se garantia a preservação de sua identidade e o direito de desistir de participar da pesquisa a qualquer momento.

\section{Resultados}

A Tabela 1 apresenta os resultados de cada uma das amostras com relação às variáveis coletadas. É possível verificar que o perfil das mães de cada amostra se mostrou significativamente diferente na maioria das variáveis avaliadas. A prevalência de DPP foi maior nas mães com parto realizado no hospital público em relação ao privado ( $26 \%$ e $9 \%$ respectivamente), enquanto a proporção de mulheres que relataram episódios anteriores de depressão foi menor no público (25\%) do que no privado (54\%). A amostra do hospital público obteve menor escore médio na Escala de Apoio Social do que a do privado (78,7 e 90,7, respectivamente) e menor média de anos de escolaridade $(9,3)$ do que no privado $(15,3)$. Verificou-se, também, que a amostra do hospital público teve menor proporção de mães que trabalhavam (40\%) do que no privado $(77 \%)$ e menor número de mães que viviam com um companheiro (81\%, no público e $99 \%$, no privado). A qualidade da vida conjugal foi julgada nada conflituosa por $85 \%$ das mulheres da amostra do hospital privado e apenas por $43 \%$ das do público.

As mães do hospital público tiveram, em relação às do privado, menor número de consultas de pré-natal em média (7,5 e 12,2, respectivamente), menor proporção de cesarianas (33\% e $77 \%$, respectivamente) e possuíam um maior número de filhos antes do parto atual. Com relação a variáveis ligadas à saúde do bebê, a amostra das mães que deram à luz no hospital público foi diferente em relação às mães do privado apenas na média dos escores APGAR do quinto minuto $(9,6$ e 9,8$)$, não tendo havido diferenças entre as médias de peso e altura dos bebês ao nascerem. Houve proporção similar de crianças do sexo masculino e feminino nas duas amostras.

Tabela 1

Caracterização das Amostras Estudadas

\begin{tabular}{|c|c|c|c|}
\hline & $\begin{array}{c}\text { Público } \\
(N=205)\end{array}$ & $\begin{array}{l}\text { Privado } \\
(N=257)\end{array}$ & $\begin{array}{l}\text { Valor } \\
\text { de } p\end{array}$ \\
\hline \multicolumn{4}{|l|}{$\overline{\text { DPP }^{1}}$} \\
\hline Sim & $26 \%$ & $9 \%$ & \multirow{2}{*}{$<0,001$} \\
\hline Não & $74 \%$ & $91 \%$ & \\
\hline \multicolumn{4}{|l|}{ Sexo do bebêt ${ }^{1}$} \\
\hline Masculino & $46 \%$ & $50 \%$ & \multirow{2}{*}{0,39} \\
\hline Feminino & $54 \%$ & $50 \%$ & \\
\hline \multicolumn{4}{|c|}{ Peso do bebê ao nascer $(\mathrm{g})^{2}$} \\
\hline Média $(D P)$ & $\begin{array}{l}3.281,4 \\
(433,6)\end{array}$ & $\begin{array}{c}3.226 \\
(454,5)\end{array}$ & 0,19 \\
\hline \multicolumn{4}{|c|}{ Altura do bebê ao nascer $(\mathrm{cm})^{2}$} \\
\hline Média $(D P)$ & $49,1(2,3)$ & $49,0(1,9)$ & 0,62 \\
\hline \multicolumn{4}{|l|}{ APGAR 5 o minuto ${ }^{4}$} \\
\hline Média $(D P)$ & $9,6(0,7)$ & $9,8(0,4)$ & $<0,001$ \\
\hline \multicolumn{4}{|l|}{ Consultas pré-natal $^{2}$} \\
\hline Média $(D P)$ & $7,5(2,2)$ & $12,2(3,4)$ & $<0,001$ \\
\hline \multicolumn{4}{|l|}{ Tipo de parto ${ }^{3}$} \\
\hline Normal & $47 \%$ & $23 \%$ & \multirow{3}{*}{$<0,001$} \\
\hline Cesariana & $33 \%$ & $77 \%$ & \\
\hline Fórceps & $20 \%$ & $1 \%$ & \\
\hline \multicolumn{4}{|c|}{ Número prévio de filhos ${ }^{4}$} \\
\hline 0 & $49 \%$ & $54 \%$ & \multirow{4}{*}{0,019} \\
\hline 1 & $31 \%$ & $40 \%$ & \\
\hline 2 & $14 \%$ & $5 \%$ & \\
\hline 3 ou mais & $5 \%$ & $0 \%$ & \\
\hline \multicolumn{4}{|l|}{ Depressão anterior ${ }^{1}$} \\
\hline Sim & $25 \%$ & $56 \%$ & \multirow{2}{*}{$<0,001$} \\
\hline Não & $75 \%$ & $44 \%$ & \\
\hline \multicolumn{4}{|l|}{ Mãe trabalha ${ }^{1}$} \\
\hline Sim & $40 \%$ & $77 \%$ & \multirow{2}{*}{$<0,001$} \\
\hline Não & $60 \%$ & $23 \%$ & \\
\hline \multicolumn{4}{|l|}{ Vive com companheiro $^{1}$} \\
\hline Sim & $81 \%$ & $99 \%$ & \multirow{2}{*}{$<0,001$} \\
\hline Não & $19 \%$ & $1 \%$ & \\
\hline \multicolumn{3}{|l|}{ Escolaridade (anos) ${ }^{2}$} & \multirow[t]{2}{*}{$<0,001$} \\
\hline Média $(D P)$ & $9,3(2,6)$ & $15,3(1,0)$ & \\
\hline \multicolumn{4}{|l|}{ Escala de Apoio Social ${ }^{4}$} \\
\hline Média $(D P)$ & $78,7(16,1)$ & $90,7(7,1)$ & $<0,001$ \\
\hline \multicolumn{4}{|c|}{ Qualidade de vida conjugal ${ }^{4}$} \\
\hline Nada conflituosa & $43 \%$ & $85 \%$ & \multirow{3}{*}{$<0,001$} \\
\hline Pouco conflituosa & $49 \%$ & $13 \%$ & \\
\hline Muito conflituosa & $8 \%$ & $1 \%$ & \\
\hline
\end{tabular}

Nota: ${ }^{1}$ Teste exato de Fisher; ${ }^{2}$ Teste t de Student $;^{3}$

Qui quadrado; ${ }^{4}$ Mann-Withney 
Para o ajuste do modelo de regressão, foram consideradas as seguintes variáveis: hospital (público/privado), número prévio de filhos, escolaridade (em anos), escore na EAS, qualidade de relação com o pai da criança (nada conflituosa, um pouco conflituosa e muito conflituosa), se vive com o companheiro (sim e não), se trabalha (sim e não) e ocorrência de depressão anterior (sim e não). Consideramos o efeito principal de cada uma dessas variáveis, bem como a interação de cada uma delas com a variável hospital. Após comparar e avaliar vários modelos através dos métodos de seleção automática Stepwise, Backward e Forward, pelo critério de comparação de modelos AIC, levando em conta também o critério da parcimônia e da significância das variáveis de cada modelo, chegou-se a um modelo com as seguintes covariáveis: qualidade da relação com o parceiro, escala de apoio social, ocorrência de depressão anterior e anos de escolaridade. Nenhuma dessas variáveis mostrou interação significativa com a variável hospital.

A Tabela 2 mostra os níveis de significância para cada uma das variáveis que compuseram o modelo final, bem como sua razão de chance com relação à presença de DPP. As análises da variável qualidade de relação com o companheiro foram feitas em função do nível nada conflituosa, a depressão anterior foi avaliada em função de sua presença versus sua ausência, enquanto o escore na EAS e a escolaridade foram considerados em seus valores absolutos para cada participante.

As razões de chance descritas na Tabela 2 indicam que, mantidas as demais variáveis fixas, mulheres com uma relação um pouco conflituosa com o pai da criança tiveram 1,96 vezes mais chance de serem diagnosticadas com DPP que mães com relação nada conflituosa. Já as mães com relação muito conflituosa tiveram 4,2 vezes mais chance de serem diagnosticadas com DPP do que mulheres sem conflitos na relação conjugal. Mulheres com relato de depressão anterior tiveram cerca de três vezes mais chances de ter DPP, mantidas as demais variáveis fixas. Cada ano de escolaridade a mais indicou uma chance $16,67 \%(1 / 0,857)$ menor de aparecimento de DPP e cada ponto a mais na EAS representou uma chance $2,77 \%(1 / 0,973)$ menor de indicação positiva para DPP. Para verificar se o modelo ajustado era adequado, foi calculada a estatística de Hosmer e Lemeshow (2000), pela qual o modelo foi considerado válido (valor de $p$ do teste $=0,19$ ).

Tabela 2

Nivel de Significância e Razão de Chance em Relação à Presença de DPP das Variáveis que Compuseram o Modelo Final de Regressão

\begin{tabular}{cccccccc}
\hline Variável & Parâmetro & Estimativa & Erro padrão & $\begin{array}{c}\text { Valor } \\
\text { de } \boldsymbol{p}\end{array}$ & Razão de chance & $\begin{array}{c}\text { Intervalo de confiança } \\
\text { da razão de chance 95\% }\end{array}$ \\
\hline $\begin{array}{c}\text { Qualidade } \\
\text { da relação }\end{array}$ & $\begin{array}{c}\text { um pouco } \\
\text { conflituosa } \\
\text { muito }\end{array}$ & 0,678 & 0,328 & 0,039 & 1,969 & 1,034 & 3,75 \\
$\begin{array}{c}\text { Escala de } \\
\text { conflituosa }\end{array}$ & 1,436 & 0,607 & 0,018 & 4,206 & 1,28 & 1,381 \\
$\begin{array}{c}\text { Apoio Social } \\
\text { Depressão } \\
\text { anterior }\end{array}$ & escore & $-0,027$ & 0,011 & 0,012 & 0,973 & 0,952 & 0,993 \\
Escolaridade & presença & $-1,112$ & 0,318 & $<0,001$ & 3,073 & 1,648 & 5,728 \\
\hline
\end{tabular}

Mediante a análise fatorial exploratória das questões da EDPE, apresentada na Tabela 3, verificou-se que foi possível agrupá-las em três fatores, responsáveis por pouco mais de $65 \%$ da variabilidade total dos dados e pelas cargas fatoriais da rotação VARIMAX. O fator 1 - sintomas depressivos - foi composto pelas questões 7, 8, 9 e 10, o fator 2 - ansiedade -, pelas questões 3, 4 e 5 e o fator 3 - anedonia - , pelas questões 1, 2 e 6, usando-se a terminologia de Mcvey e Tuohy (2007).

Tabela 3

Resultado da Análise Fatorial Confirmatória das Questões da EDPE

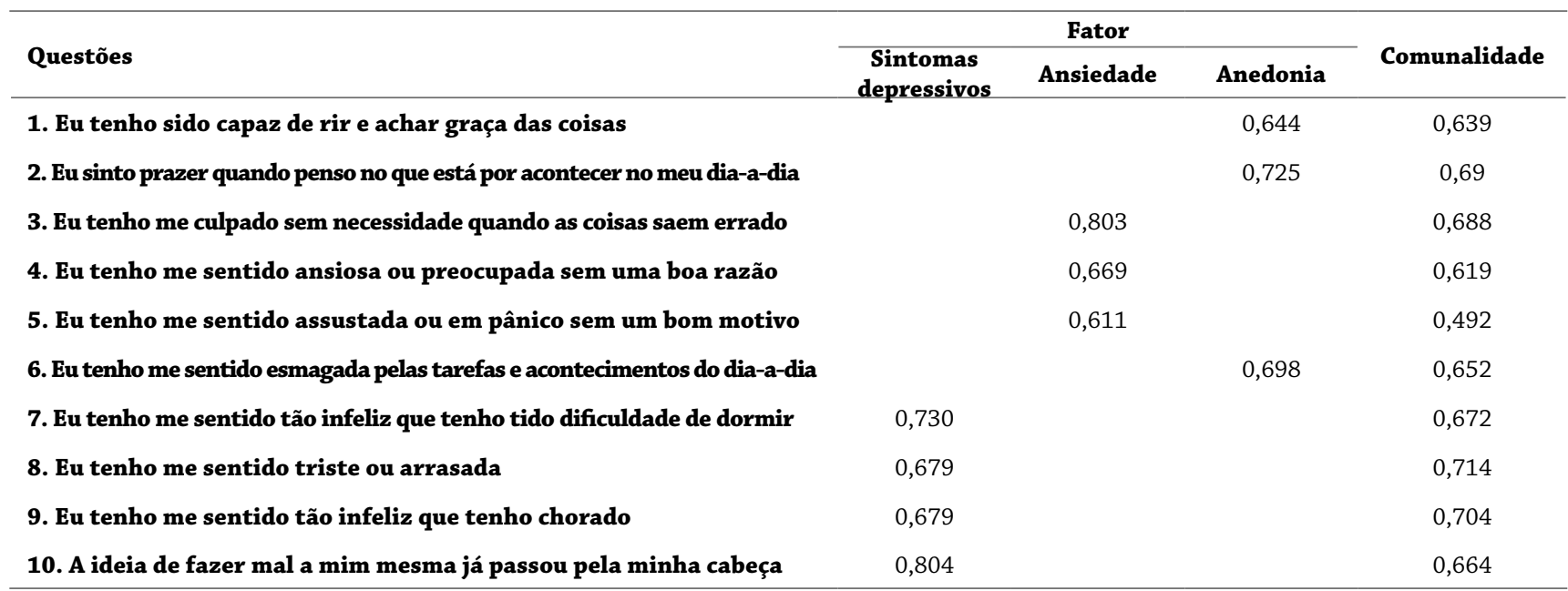

Nota: Fator de carga: rotação Varimax com Kaizer normalization 
O modelo teórico de equações estruturais testado, ilustrado na Figura 1, foi composto de 21 variáveis: as 10 questões da EDPE, as oito variáveis tomadas como possivelmente preditoras dos três fatores latentes da EDPE e as três variáveis latentes, formadas justamente pelos três fatores da análise fatorial exploratória anterior. Após análise do modelo e eliminação das variáveis que não mantinham as relações significativas propostas inicialmente, chegouse ao modelo final da Figura 1b, composto por seis componentes preditores, três latentes e 10 questões da EDPE, totalizando 19 variáveis. O modelo mostrou-se bem ajustado, com valor do RMSEA de 0,08 e todas as demais métricas (NFI, IFI, RFI, TLI, GFI e AGFI) maiores do que 0,87 .

Figura 1. Modelos de Equações Estruturais Teórico (a) e Final (b), com as Respectivas Estimativas Padronizadas

Modelo teórico (a)

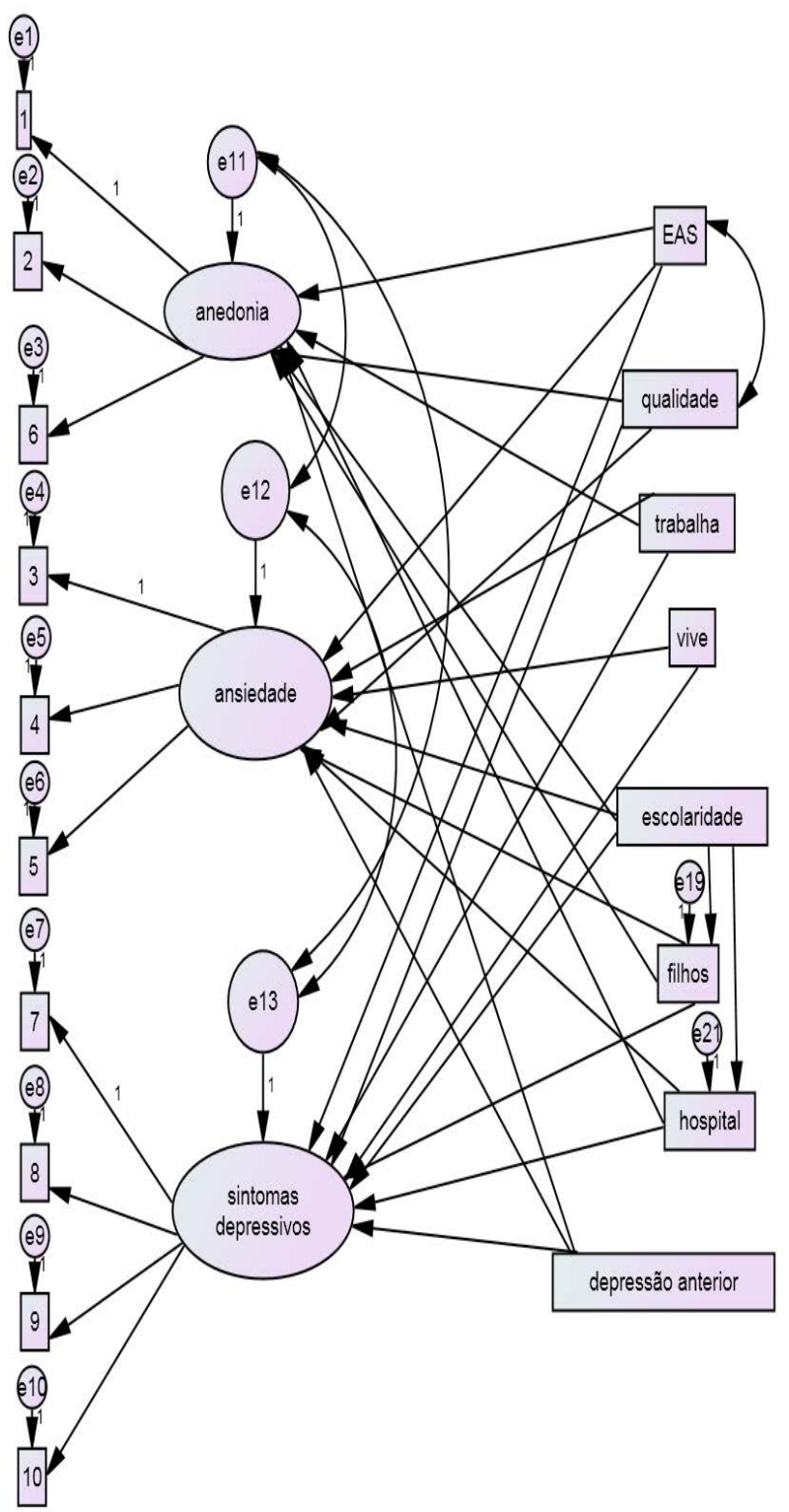

Todas as relações existentes no modelo final foram significativas nas direções propostas e os valores das estimativas padronizados constam da tabela 4. Diferentemente do modelo teórico proposto, verificou-se, no modelo final, que apenas as variáveis apoio social, qualidade de vida com o companheiro e presença de depressão anterior apresentaram relações significativas com os três fatores encontrados na EDPE. A escolaridade e o hospital se relacionaram apenas com os sintomas depres-
Modelo final (b)

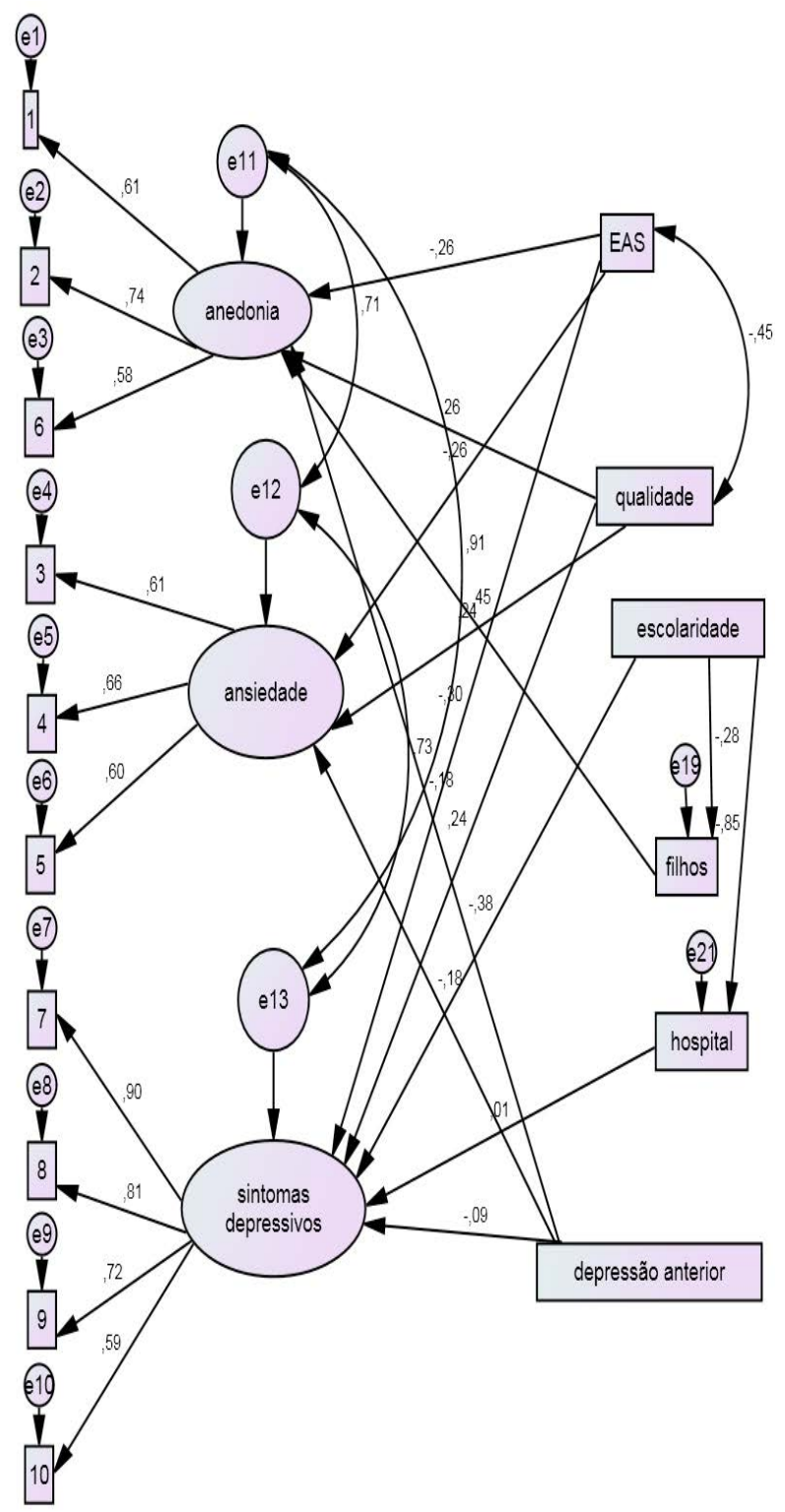

sivos e o número prévio de filhos mostrou relação significativa apenas com o fator anedonia. Entre as variáveis observáveis, foi ainda possível verificar a influência da escolaridade sobre o hospital e número prévio de filhos e uma correlação negativa entre o escore na EAS e o nível de conflito com o companheiro.

O modelo final apresenta uma análise fatorial confirmatória, mostrando que existe a relação entre os fatores anedonia, ansiedade e sintomas depressivos, variáveis latentes, com 
as respectivas questões da EDPE, conforme descrito na análise exploratória anterior. Por questão de identificabilidade estatística, assumiu-se que as questões 1, 3 e 7 da EDPE tinham peso de regressão 1; as estimativas padronizadas delas e das outras questões da escala estão na Tabela 4.

O modelo indica a complexidade das relações envolvidas, mas podemos discutir o peso de cada uma, tomando-se como parâmetro todas as demais se mantendo em valores constantes. Entre as variáveis preditivas observáveis e os fatores latentes, a relação com maior estimativa de peso de regressão em módulo encontrada foi a da escolaridade com os sintomas depressivos $(-0,374)$, indicando que quanto maior a escolaridade, menores os sintomas depressivos. Em seguida, temos as estimativas das relações da variável qualidade da relação com o companheiro, indicando que quanto pior a relação, maior o valor das latentes anedonia, ansiedade e sintomas depressivos, com estimativas de pesos de regressão de 0,286, 0,251 e 0,210, respectivamente. O modelo mostrou que quanto maior o escore na EAS, menores os valores dos três fatores, com estimativas de - 0,207 com a anedonia, -0,250 com a ansiedade e -0,219 com os sintomas depressivos. A ausência de depressão anterior também parece afetar os valores das três variáveis latentes, com pesos de $-0,190$ com a anedonia, $-0,203$ com a ansiedade e $-0,166$ com os sintomas depressivos. O maior número de filhos anteriores à presente gravidez influenciou positivamente a anedonia, com peso estimado em 0,131 e o hospital influenciou a presença de sintomas depressivos com peso de -0,149, sendo que a relação negativa indica diminuição nos sintomas das mães que tiveram seus filhos no hospital privado.

O modelo também evidenciou relação entre as variáveis observáveis escolaridade com número de filhos e hospital. As relações indicam que quanto maior a escolaridade, menor o número prévio de filhos, mantidas as demais variáveis constantes, com estimativa de peso de regressão de -0,848 e maior a chance de a mãe estar no hospital privado, com peso de -0,219. Foi possível verificar, ainda, a correlação entre a variável apoio social e a qualidade de relacionamento com o parceiro, com estimativa de $-0,454$.

Tabela 4

Pesos de Regressão Padronizados para as Relações Causais do Modelo de Equações Estruturais

\begin{tabular}{|c|c|c|c|c|c|}
\hline Efeito & & Causa & $\begin{array}{c}\text { Estimativa de } \\
\text { peso de regressão }\end{array}$ & Erro padrão & Valor de $p$ \\
\hline Variável observável & & Variável observável & & & \\
\hline Hospital & $<--$ & escolaridade & $-0,219$ & 0,004 & $<0,001$ \\
\hline Número de filhos & $<--$ & escolaridade & $-0,848$ & 0,01 & $<0,001$ \\
\hline Variável Latente & & Variável Observável & & & \\
\hline Anedonia & $<---$ & EAS & $-0,207$ & 0,002 & $<0,001$ \\
\hline Anedonia & $<--$ & Número de filhos & 0,131 & 0,028 & 0,002 \\
\hline Anedonia & $<---$ & Qualidade da relação & 0,286 & 0,052 & $<0,001$ \\
\hline Anedonia & $<---$ & Depressão anterior & $-0,190$ & 0,052 & $<0,001$ \\
\hline Ansiedade & $<--$ & EAS & $-0,250$ & 0,003 & $<0,001$ \\
\hline Ansiedade & $<---$ & Qualidade da relação & 0,251 & 0,065 & $<0,001$ \\
\hline Ansiedade & $<---$ & Depressão anterior & $-0,203$ & 0,064 & $<0,001$ \\
\hline Sintomas depressivos & $<---$ & EAS & $-0,219$ & 0,002 & $<0,001$ \\
\hline Sintomas depressivos & $<--$ & Escolaridade & $-0,374$ & 0,011 & $<0,001$ \\
\hline Sintomas depressivos & $<---$ & Qualidade da relação & 0,210 & 0,055 & $<0,001$ \\
\hline Sintomas depressivos & $<---$ & Depressão anterior & $-0,166$ & 0,054 & $<0,001$ \\
\hline Sintomas depressivos & $<---$ & Hospital & $-0,149$ & 0,077 & 0,023 \\
\hline Variável Observável & & Variável Latente & & & \\
\hline Q1_EDPE & $<--$ & Anedonia & 0,74 & & \\
\hline Q2_EDPE & $<---$ & Anedonia & 0,699 & 0,083 & $<0,001$ \\
\hline Q6_EDPE & $<---$ & Anedonia & 0,539 & 0,1 & $<0,001$ \\
\hline Q3_EDPE & $<---$ & Ansiedade & 0,58 & & \\
\hline Q4_EDPE & $<---$ & Ansiedade & 0,692 & 0,126 & $<0,001$ \\
\hline Q5_EDPE & $<---$ & Ansiedade & 0,601 & 0,105 & $<0,001$ \\
\hline Q7_EDPE & $<--$ & Sintomas depressivos & 0,752 & & \\
\hline Q8_EDPE & $<--$ & Sintomas depressivos & 0,847 & 0,064 & $<0,001$ \\
\hline Q9_EDPE & $<--$ & Sintomas depressivos & 0,846 & 0,055 & $<0,001$ \\
\hline Q10_EDPE & $<--$ & Sintomas depressivos & 0,596 & 0,041 & $<0,001$ \\
\hline \multicolumn{6}{|l|}{ Correlação } \\
\hline EAS & $\langle--\rangle$ & Qualidade da relação & $-0,454$ & 0,372 & $<0,001$ \\
\hline
\end{tabular}




\section{Discussão}

Como se observa pela Tabela 1 , foram grandes as diferenças entre as amostras, exceto quanto às características dos bebês ao nascer. As diferenças dizem respeito a fatores psicossociais e sociodemográficos, que ilustram com clareza modos de vida diferentes entre as classes sociais a que as participantes pertenciam. As mulheres de nível socioeconômico baixo tinham maior número de filhos, menos anos de escolaridade e, entre elas, foi menor a prevalência das que disseram ter trabalho. Entre essas mulheres, que deram à luz no hospital público, foi menor a prevalência das que viviam com um companheiro, e aquelas que tinham parceiro relataram relações mais conflituosas com ele, o que, em parte, contribuía para que se sentissem menos amparadas socialmente. Estes dados, com pequenas diferenças, são confirmados em todos os estudos que investigaram a influência de variáveis semelhantes (e.g. Aliane et al., 2011; Cruz et al., 2005; Halbreich \& Karkun, 2006; Miyake et al., 2001; Segre et al., 2007; Silva et al., 2012).

Do ponto de vista de atenção à saúde, ressalta-se o maior número de consultas de pré-natal entre as mulheres de classes mais altas e, sobretudo, o grande número de cesarianas no hospital privado: mais que o dobro do encontrado em hospital público. Este dado é particularmente alarmante, tendo em vista que a literatura relata maiores danos à saúde de mulheres que tiveram cesarianas planejadas do que as que tiveram partos normais planejados (e.g. Liu et al., 2007). Este é um tema que merece ser muito bem investigado em trabalhos posteriores.

Embora a prevalência da DPP, quando analisada isoladamente de outras variáveis, tenha se revelado significativamente maior entre as mães do hospital público, após ajuste do modelo, que incluiu apenas as variáveis psicossociais e sociodemográficas, verificou-se efeito significativo sobre a DPP apenas das variáveis nível de escolaridade, escore obtido na Escala de Apoio Social, qualidade do relacionamento com o parceiro e ocorrência de depressão em algum momento anterior à gestação ou ao puerpério. Portanto, a variável nível socioeconômico, representada pelo tipo de hospital, ficou fora do modelo. A escolaridade sobressai neste estudo como principal indicador sociodemográfico, diferentemente do que encontraram Moraes et al. (2006), Segre et al. (2007) e Silva et al. (2012), que constataram que baixa renda é um forte preditor da ocorrência DPP. Contudo, Ruschi et al. (2007) também verificaram que quanto mais baixa a escolaridade da mãe maior a prevalência de DPP. Já Miyake et al. (2001) não encontraram influência de renda nem de escolaridade sobre o risco de desenvolver depressão. A respeito das diferenças entre a influência de escolaridade e nível de renda sobre o desfecho, estudo de Muller (2002) apresentou achado semelhante ao encontrado no presente estudo. $\mathrm{O}$ autor encontrou que falta de escolaridade superior explicava o efeito de desigualdade social e era um poderoso preditor de mortalidade geral nos EUA.

Extrapolando os dados de Muller (2002) para a DPP, constatamos, no presente estudo, que a grande maioria das gestantes que deram à luz no hospital privado tinha nível superior de escolaridade, enquanto mulheres com esse nível de escolaridade foram exceção no hospital público. O fato de não se verificar efeito de nível socioeconômico após ajuste do modelo, neste e em outros estudos, pode ser atribuído ao grande número de variáveis envolvidas e à complexidade de sua relação com a depressão pós-parto.

O achado de que o apoio social é fator protetor para a DPP confirma os dados de inúmeros estudos, realizados tanto no Brasil (Cruz et al., 2005; Mello Jr. et al., 2012, Silva et al., 2012) quanto em outros países (Chandran et al., 2002.; Topiwala et al., 2012). Com respeito à avaliação da qualidade da relação com o pai da criança, aferida pelo nível de conflito percebido pelas participantes, nossos dados também confirmam estudos que indicam que relações conflituosas são fator preditivo de falta de apoio social e de DPP (Mamun et al., 2009).

Da mesma forma, o achado de que um histórico prévio de depressão/ansiedade/ tristeza é preditivo da ocorrência de DPP é confirmado por diversos trabalhos (Chandran et al., 2002; Quelopana et al., 2011; Ruschi et al., 2007). Uma análise original deste trabalho diz respeito às subescalas encontradas na EDPE. Os fatores encontrados neste estudo confirmam os constatados por Mcvey e Tuohy (2007), com exceção de um dos itens (5) que, em nossa análise, foi incluído como relacionado com a anedonia e que foi excluído na análise daqueles autores.

O modelo indica que a depressão anterior associou-se aos três fatores da EDPE: ansiedade, sintomas de depressão e anedonia. Esse resultado reforça os diversos estudos que apontam depressão prévia como fator fortemente preditor de DPP. A pontuação na escala de apoio social (EAS) também se relacionou com os três fatores, corroborando seu peso como fator de risco para quadros depressivos.

A relação entre apoio social e nível de conflito com o parceiro confirma achados de estudos que revelam que o apoio do parceiro é fundamental como elemento de apoio social (e.g. Cruz et al., 2005). O número de filhos relacionou-se com escolaridade e anedonia. A variável tamanho da família, quando comparada a outros fatores de risco e isolada de determinantes socioeconômicos e culturais, tem sido pouco explorada em relação à DPP e merece aprofundamento em estudos posteriores.

A complexidade do modelo e das relações confirma que a escala não é unidimensional como indicaram Mcvey e Tuohy (2007). Tal constatação pode ser útil para a compreensão dos sintomas que caracterizam a depressão pós-parto e dos mecanismos subjacentes, favorecendo, assim, a adequação de propostas de prevenção e tratamento. O detalhamento do modelo em sua complexidade enseja diversas discussões e consequências que merecem um trabalho à parte. Por exemplo: é fundamental tratar a depressão previamente e durante a gestação; ajudar a mulher a inserir-se em uma rede de apoio social e trabalhar o relacionamento conjugal poderá protegê-la de alguns dos fatores envolvidos na depressão.

Do ponto de vista de políticas públicas com efeito preventivo, chama a atenção o dado de que uma educação formal de maior duração e mais completa é um fator protetor para a depressão pós-parto, sendo essa mais uma evidência no sentido de que a educação formal precisa ser incentivada e aprimorada no Brasil. No que diz respeito às políticas de saúde, os resultados que mostram que um apoio social mais efetivo é um importante fator protetor para a DPP indicam a importância do incentivo da adoção, por parte dos serviços de atenção pri- 
mária em saúde, de práticas preventivas e terapêuticas e de apoio às gestantes e puérperas - com especial atenção para aquelas com histórico de depressão prévia.

É importante ressaltar uma limitação metodológica do presente estudo, que diz respeito à forma de coleta de dados e que pode ter gerado algum viés nos resultados. Enquanto as mulheres do hospital público foram recrutadas na Unidade Básica de Saúde e a EDPE foi aplicada pessoalmente, as mães do hospital privado foram recrutadas no próprio hospital, após o parto, e foram entrevistadas por telefone.

\section{Referências}

Aliane, P. P., Mamede, M. V., \& Furtado, E. F. (2011). Revisão sistemática sobre fatores de risco associados à depressão pós-parto. Psicologia em Pesquisa, 5(2), 146-155. Recuperado de http://pepsic.bvsalud.org/scielo.php?script=sci_arttext\&pid=S1982-12472011000200007\&lng=pt\&tlng=pt

Arbuckle, J. L. (1995). IBM SPSS 19. User's Guide. Recuperado de www.amosdevelopment.com/download/amos.pdf

Camacho, R. S., Cantinelli, F. S., Ribeiro, C. S., Cantilino, A., Gonsales, B. K., Braguittoni, E., \& Rennó Jr., J. (2006). Transtornos psiquiátricos na gestação e no puerpério: classificação, diagnóstico e tratamento. Archives of Clinical Psychiatry, 33(2), 92-102. doi: 10.1590/S010160832006000200009

Cantilino, A., Zambaldi., C. F., Sougey. E. B., \& Rennó Jr, J. (2010). Transtornos psiquiátricos no pós-parto. Revista de Psiquiatria Clínica, 37(6), 278-284. doi: 10.1590/S0101-60832010000600006

Chandran, M., Tharyan, P., Muliyil, J., \& Abraham, S. (2002). Post-partum depression in a cohort of women from a rural area of Tamil Nadu, India: Incidence and risk factors. British Journal of Psychiatry, 181(6), 499-504. doi: 10.1192/bjp.181.6.499

Cox, L., Holden, J. M., \& Sagovsky, R. (1987). Detection of postnatal depression: development of the 10-item Edinburgh Postnatal Depression Scale. British Journal of Psychiatry, 150(6), 782-786. doi: 10.1192/ bjp.150.6.782

Cruz, E. B. S., Simões, G. L., \& Faisal-Cury, A. (2005). Rastreamento da depressão pós-parto em mulheres atendidas pelo programa de saúde da família. Revista Brasileira de Ginecologia e Obstetrícia, 27(4), 181-188. doi: 10.1590/S0100-72032005000400004

Da-Silva, V. A., Moraes-Santos, A. R., Carvalho, M. S., Martins, M. L. P., \& Teixeira, N. A. (1998). Prenatal and postnatal depression among low income Brazilian women. Brazilian Journal of Medical and Biological Research, 31(6), 799-804. doi: 10.1590/S0100-879X1998000600012

Ertel, K. A., Koenen, K. C., Rich-Edwards, J. W., \& Gillman, M. W. (2010), Maternal depressive symptoms not associated with reduced height in young children in a US Prospective Cohort Study. PLoS/ ONE; 5(10):e13656. doi: 10.1371/journal.pone.0013656

Gaynes, B. N., Gavin, N., Meltzer-Brody, S., Lohr., K. N., Swinson., T., Gartlehner., G...., Miller, W. C. (2005). Perinatal depression: Prevalence, screening accuracy, and screening outcomes. AHRQ Evidence Report Summaries. Rockville (MD): Agency for Healthcare Research and Quality (US). Recuperado de: http://www.ahrq.gov/clinic/epcsums/peridepsum.htm.

Griep, R. H., Chor, D., Faerstein, E., Werneck, G. L., \& Lopes, C. S. (2005). Validade de constructo de escala de apoio social do Medical Outcomes Study adaptada para o português no Estudo Pró-Saúde. Cadernos de Saúde Pública, 21(3), 703-714. doi: 10.1590/S0102-311X2005000300004

Grote, V., Vik, T., von Kries, R., Luque, V., Socha, J., Verduci, E., ... Koletzko., B. (2010). Maternal postnatal depression and child growth: A European Cohort Study. BMC Pediatrics, 10(14). doi: 10.1186/1471-2431-10-14

Halbreich, U., \& Karkun, S. (2006). Cross-cultural and social diversity of prevalence of postpartum depression and depressive symptoms. Journal of Affective Disorders, 91(2-3), 97-111. doi: 10.1016/j.jad.2005.12.051

Hosmer, D., \& Lemeshow, S. (2000). Applied logistic regression ( $2^{\mathrm{a}}$ ed.). Nova Iorque: John Wiley \& Sons.

Klainin, P., \& Arthur , D .G. (2009). Postpartum depression in Asian cultures: A literature review. International Journal of Nursing Studies, 46(10), 1355-73. doi: 10.1016/j.ijnurstu.2009.02.012
Liu, S. , Liston, R. M., Joseph, K. S., Heaman, M., Sauve, R., \& Kramer, M. S. (2007). Maternal mortality and severe morbidity associated with low-risk planned cesarean delivery versus planned vaginal delivery at term. Canadian Medical Association Journal, 176(4), 455-460. doi: 10.1503\%2Fcmaj.060870

Mamun, A. A., Clavarino, A. M., Najman, J. M., Williams, G. M., O'Callaghan M. J., \& Bor, W. (2009). Maternal depression and the quality of marital relationship: A 14-year prospective study. Journal of Women's Health, 18(12), 2023-31. doi: 10.1089/jwh.2008.1050

Mcvey, C., \& Tuohy, A. (2007). Differential effects of marital relationship and social support on three subscales identified within the Edinburgh Postnatal Depression Scale. Journal of Reproductive and Infant Psychology, 25(3), 203-9. doi: 10.1080/02646830701467256

Melo Jr., E. F., Cecatti, J. G., Pacagnella, R. C., Leite, D. F. B., Vulcani, D. E., \& Makuch, M. Y. (2012). The prevalence of perinatal depression and its associated factors in two different settings in Brazil. Journal of Affective Disorders, 136(3), 1204-8. doi: 10.1016/j.jad.2011.11.023

Miyake, Y., Tanaka, K., Sasaki, S., \& Hirota, Y. (2001). Employment, income, and education and risk of postpartum depression: The Osaka maternal and child health study. Journal of Affective Disorders, 130(3), 133-7. doi: 10.1016/j.jad.2011.11.023

Moraes, I. G. S., Pinheiro, R. T., Silva, R. A., Horta B. L., Sousa, P. L. R., \& Faria, A. D. (2006). Prevalência da depressão pós-parto e fatores associados. Revista de Saúde Pública, 40(1), 65-70. doi: 10.1590/S003489102006000100011

Muller, A. (2002). Education, income inequality, and mortality: A multiple regression analysis. British Medical Journal, 324(7328), 1-4. doi: 10.1136/ bmj.324.7328.23

Norusis, M. (2011). IBM SPSS Statistics 19 Guide to Data Analysis. Recuperado de http://free-download-books-medical-education.com/131-ibm-spss-statistics-19-guide-to-data-analysis.html

Organização Mundial da Saúde (2007). CID-10 Classificação Estatística Internacional de Doenças e Problemas Relacionados à Saúde (10ª ed.). São Paulo: EDUSP.

Quelopana, A. M., Champion, J. D., \& Rubilar, T. R. (2011). Factors associated with postpartum depression in Chilean women. Health Care Women International Journal, 32, 939-49. doi: 10.1080/07399332.2011.603866

Ruschi, G. E. C., Sun, S. Y., Mattar, R., Chambô Filho, A., Zandonade, E., \& Lima, V. J. (2007). Aspectos epidemiológicos da depressão pós-parto em amostra brasileira. Revista de Psiquiatria do Rio Grande do Sul, 29(3), 27480. doi: 10.1590/S0101-81082007000300006

Santos, C. M. T., Almeida, G. O., \& Souza, T. S. (2009). Depressão pós-parto revisão da literatura. Psicologia em Foco, 3(2), 1-1. Recuperado de http:// linux.alfamaweb.com.br/sgw/downloads/161_014747_Formatado1 Depressaopos-parto.pdf

Santos, I. S., Matijasevich, A., Tavares, B. C., Barros, A. J., Botelho, I. P., Lapolli, C., ... Barros, F. C. (2007).Validation of the Edinburgh Postnatal Depression Scale (EPDS) in a sample of mothers from the 2004 Pelotas Birth Cohort Study. Cadernos de Saúde Pública, 23(11), 2577-2588. doi: 10.1590/S0102-311X2007001100005

Santos, M. F. S, Martins, F. C, \& Pasquali, L. (1999). Escalas de auto-avaliação de depressão pós-parto: estudo no Brasil. Revista de Psiquiatria Clínica 26(2), 32-40. Recuperado de http://www.hcnet.usp.br/ipq/revista/

Segre, L. S., O'Hara, M. W., Arndt, S., \& Stuart, S. (2007). The prevalence of postpartum depression. The relative significance of three social status indices. Social Psychiatry and Psychiatric Epidemiology, 42(4), 316-321.

Silva, E. T., \& Botti, N. C. L. (2005). Depressão puerperal - uma revisão de literatura. Revista Eletrônica de Enfermagem, 7(2), 231-238. Recuperado de http://www.fen.ufg.br.

Silva, R., Jansens, K., Souza, L., Quevedo, L., Barbosa, L., Moraes, I, ... Pinheiro, R. (2012). Sociodemographic risk factors of perinatal depression A cohort study in the public health care system. Revista Brasileira de Psiquiatria, 34(2), 143-148. doi: 10.1590/S1516-44462012000200005

Topiwala, A., Hothi, G., \& Ebmeier, K. P. (2012). Identifying patients at risk of perinatal mood disorders. Practitioner, 256(1751), 15-18.

Nota:

1. Pesquisa financiada pela Fundação de Amparo à Pesquisa do Estado de São Paulo (processo no 06/59192-2) 
Maria de Lima Salum e Morais, Doutora em Psicologia pelo Departamento de Psicologia Experimental (PSE) do Instituto de Psicologia da USP (IPUSP), é Pesquisadora científica V do Instituto de Saúde (IS) da Secretaria de Estado de Saúde de São Paulo (SES). Endereço para correspondência: Rua Novo Cancioneiro, 75 - ap. 31, CEP: 04704-080 - São Paulo, SP. E-mail: salum@isaude.sp.gov.br

Luiz Augusto Marcondes Fonseca, Doutor em Saúde Pública, área de Epidemiologia, Universidade de São Paulo, Faculdade de Saúde Pública (FSP-USP). Pós-doutor pela University of California, Berkeley, é Pesquisador associado, Departamento de Medicina Preventiva, Faculdade de Medicina da Universidade de São Paulo

(FMUSP). E-mail: luizaugusto.fonseca@hc.fm.usp.br

Vinicius Frayze David, Mestre em Psicobiologia pela Universidade de São Paulo, Faculdade de Filosofia, Ciências e Letras de Ribeirão Preto FFCLRP-USP, é Especialista em Laboratório no Instituto de Psicologia da Universidade de São Paulo IPUSP. E-mail: viniciusfdavid@gmail.com

Lia Matos Viegas, Mestre em Psicologia Experimental pelo IP-USP, é doutoranda do Instituto de Psicologia da Universidade de São Paulo (IP-USP). E-mail: lia.viegas@usp.br

Emma Otta, Professora titular do Departamento de Psicologia Experimental (área de Etologia), é Chefe do Departamento de Psicologia Experimental do Instituto de Psicologia da USP. E-mail: emmaotta@gmail.com 